\title{
THE INFLUENCE OF PHYTOTHERAPY ON PROLACTIN LEVEL IN MACROPROLACTINOMA PATIENTS
}

\author{
Ivo Trogrlić ${ }^{1}$, Dragan Trogrlić*1 and Zoran Trogrlić ${ }^{1}$ \\ "DREN" Ltd, Žepče, Bukovik 3, 72230 Žepče, B \& H \\ Bosnia \& Herzegovina \\ *E-mail: dragan.trogrlic@tel.net.ba
}

\begin{abstract}
The study aims at demonstrating the efficiency of phytotherapy in regulation of prolactin levels in patients diagnosed with pituitary macroprolactinoma. The study made use of workup outcomes submitted by treating healthcare facilities where the patients were first diagnosed with macroprolactinomas based on diagnostic imaging (MRI and/or CT), laboratory workup, and hormone status estimation. The data in reference served as the baseline for a comparative follow-up of phytotherapeutic efficiency in terms of comparison of medical data obtained prior to phytotherapy and those submitted following herbal remedy use. The study displays the results of a five-year follow-up of macroprolactinoma patients undergoing phytotherapy. In well-responding patients, the benefit of phytotherapy in terms of prolactin level putdown can be seen 3-5 months following the phytotherapy commencement. A special attention should be paid to the favourable results obtained with patients who chose to resort to phytotherapy only. The primary outcome measure used to monitor the efficiency of the administered phytotherapy was prolactin level determined prior to, during the course of, and following phytotherapy. Implementation of phytotherapy to the effect of prolactin level regulation in patients diagnosed with macroprolactinoma, represents a completely novel therapeutic approach. The majority of the diseased resorted to phytotherapy once they were left out of any other therapeutic option offered by their treating healthcare facilities. This fact gives a substantial rise to the significance of the results presented herein and justifies the role of phytotherapy in macroprolactinoma-induced hyperprolactinaemia treatment.
\end{abstract}

Key words: phytotherapy; macroprolactinoma; prolactin; pituitary gland.

\section{Introduction}

In physiological milieu, prolactin hormone level (PRL) normally rises during pregnancy and lactation. Any other increase in prolactin levels should be viewed as a sign of pathology (Mah and Webster, 2001). Apart from iatrogenic hyperprolactinaemia induced by certain dopamine receptor-binding drugs, the second most common cause of an enhanced prolactin release are benign pituitary tumours - prolactinomas (Horvath and Kovacs, 1992). The latter are essentially secretion tumours stemming from lactotrophic pituitary cells primarily in service of prolactin synthesis and release. Prolactinomas manage to preserve the characteristics of their "parental" tissue, so that the diseased experience a more or less pronounced hyperprolactinaemia (Guyton and Hall, 2003; Mah and Webster, 2001).

Based on their size, prolactinomas can be divided into micro- and macroprolactinomas. Prolactinomas can be differentiated based not only on their magnitude, but on their secretory potential as well (Asa, 1988). Most commonly, the larger the tumour, the higher the hyperprolactinaemia, so that in case of microprolactinoma a moderate rise in prolactin levels is to be expected, while in case of macroprolactinoma prolactin levels may rise a few tens, or even a few hundred folds above the referent values (Asa, 1988; Levy and Lightman, 1993). To the effect of treating these tumours, contemporary medicine resorts to pharmacotherapy, surgical treatment and radio-therapy (Braucks et al, 2003).

This study aims at demonstrating the efficiency of phytotherapy in diminishing macroprolactinoma's secretory potential and the possible use of phytotherapy (PT) in macroprolactinoma-induced hyperprolactinaemia regulation.

\section{Subjects and Methods}

The study was carried out within 2005-2009 timeframe, and was grounded on earlier notions on therapeutic impact of PT on infertility. The study comprised a total of 30 patients diagnosed with macroprolactinomas, out of which 19 (63.4\%) women and $11(36.6 \%)$ men. The patients were aged 16 to 78 , i.e. 38.8 on the average. Prior to the phytotherapy (PT) commencement, the patients provided medical records in proof of their diagnosis made based on magnetic resonance imaging (MRI) and/or computed tomography (CT) of the affected site, as well as based on hormone status determination and field-ofvision testing. The data in reference served as the baseline for the comparative monitoring of PT efficiency, in terms of mutual comparison of data entered into medical records prior to PT and those estimated following herbal remedy use.

Patients diagnosed with macroprolactinoma had been treated with three types of herbal preparations (Preparation 1, Preparation 2, and Preparation 3), mutually differing in their composition. All of the three comprise a mixture of pounded herbal fragments and lack any other additives whatsoever. Plant ingredients are pounded to the standard degree (Kovačević, 2000; Lukić, 1993). The herbal remedies in question are prepared in form of teas to be consumed on an everyday basis in regular time intervals, once a day each, conformant to the following timeline: Preparation 1 at 7:00 am, Preparation 2 at 2:00 
$\mathrm{pm}$, and Preparation 3 at 8:00 pm. All three are prepared in the same manner, a single tea dose thereby calling for $1.5 \mathrm{~g}$ of herbal mixture and $200 \mathrm{~cm}^{3}$ of water.

Following PRL estimation prior to and following PT, completed in all 30 patients, the latter were divided into two groups. Group 1 consisted of 22 patients administered not only PT, but pharmacotherapy (dopamine agonists) as well (herein after referred to as: the DA \& PT arm). As for the gender, the group in reference was made up of $12(54.5 \%)$ women and 10 (45.5\%) men, their age spanning from 16 to 78 (40.7 on the average).

Group 2 consisted of 8 patients administered PT only (herein after referred to as: the PT arm). The group in reference embraced $7(87.5 \%)$ women and one (12.5\%) man, their age spanning from 20 to 49, i.e. 33.5 on the average.

Within the frame of the follow-up process, the following outcome measures and records were of interest:

- $\quad$ Prolactin levels (ng/mL) established prior to and following PT in all 30 patients;

- $\quad$ Records of patients concurrently treated with PT and dopamine agonists (22 patients);

- $\quad$ Records of patients on PT only (8 patients).

In addition to the aforementioned indicators, records of some other parameters (e.g. prior treatments, tumour size, PT commencement and duration, patient age and gender, recurrences, and so forth) had been kept as well.

Data on the parameters detailed above posed as the baseline for mathematical-statistical analysis aimed at corroborating or rejecting certain hypotheses on PT outcomes. In order to reach as reliable conclusions as possible, the analysis of changes in PRLs established prior to and following PT was preceded by the analysis of baseline data credibility. Nevertheless, the primary goal of the analyses completed within this frame was to verify PT efficiency. To that effect, the hypothesis on the existence of significant differences in pre- and post-PT PRLs was tested at the significance levels of 5\%, $1 \%$, and $0.1 \%$ (exceptionally also at the significance level above 5\% in cases where information on PT efficiency was short). To the aforementioned goal, not only standard, but also non-parametric statistical methods were used. Namely, the sample size and the statistical parameters obtained (central values, ranges and metric output dispersion) clearly indicated that parametric, i.e. "classical" statistical methods would be an inappropriate choice. Therefore, statistical evaluation primarily made use of non-parametric methods capable of accurately testing the hypothesis independent of data distribution. The following tests were employed:

- Wilcoxon matched-pairs signed-rank test

- $\quad$ Kruskal-Wallis test

- Wilcoxon signed-rank test

- Mann-Whitney U test (rank-sum test)

The results obtained by virtue of the statistical methods quoted above, were further verified using the programme package IBM SPSS Statistics 18 (test version), as well as using the free software environment $\mathbf{R}$ Statistics, version 2.11.1, that serves the purposes of statistical computing and graphics. Graphic interpretation of PT outcomes represents an integral part of the hypothesis testing process.

\section{Results \\ Analysis of PRL changes witnessed prior to and following PT - the entire patient sample}

In order to asses the PT efficiency, PRL changes were first analysed in all study patients. Prior to the PT commencement, the levels of this hormone were increased in 29 patients, while the remaining male patient had his PRLs within normal boundaries; however, he decided to undergo PT due to other tumour-induced symptoms. Primary PRL indicators obtained using descriptive statistics, point towards significant differences in PRL means registered prior to and following PT. The mean PRL recorded in the entire patient sample prior to the PT commencement equalled to 245.1, while the one seen following PT equalled to $30.5 \mathrm{ng} / \mathrm{mL}$. In other words, post-PT PRL mean was decreased by $214.6 \mathrm{ng} / \mathrm{mL}$, i.e. 87.7\%. In this particular case, the statistical significance of the decrease in PRL level was tested at the significance level of $1 \%$.

The assumed PT efficiency was corroborated by testing the hypothesis on PRL means prior to and following PT, done at the significance levels of 5\% and 1\%. Namely, the differences in PRLs seen prior to and following PT reveal a significant decrease in PRLs determined following the PT completion. More precisely, it can be stated with 95\% and even 99\% certainty (and the pertaining risk of error of 5\% and 1\%, respectively) that the mean PRLs seen in the PT arm following PT completion are significantly lower as compared to those registered prior to PT commencement. It can be easily proven that the above statement applies also for $0.1 \%$-significance level, as corroborated by an adequate hypothesis testing. For visual display of the results discussed above, please refer to Figure 1.

\section{Group 1: Changes in PRLs seen in the DA \& PT arm}

At the PT launch point, a total of 22 patients had already been administered with pharmacotherapy (in form of dopamine agonists, DAs); the patients in reference continued to use these drugs as prescribed throughout the PT period. PRL means obtained by virtue of descriptive statistics prior to and following PT, viewed as the primary outcome measure, revealed statistically significant changes in PRL means seen following the PT completion. Prior to the PT commencement, the PRL mean of $189.4 \mathrm{ng} / \mathrm{mL}$ was registered, while the correspondent value registered following PT completion equalled to $27.5 \mathrm{ng} / \mathrm{mL}$. The difference in pre- and post-PT PRL means equals to $161.9 \mathrm{ng} / \mathrm{mL}$, disclosing an 85.5\%-PRL decrease. 
Trogrlic et al., Afr J Tradit Complement Altern Med. (2012) 9(1):67-72

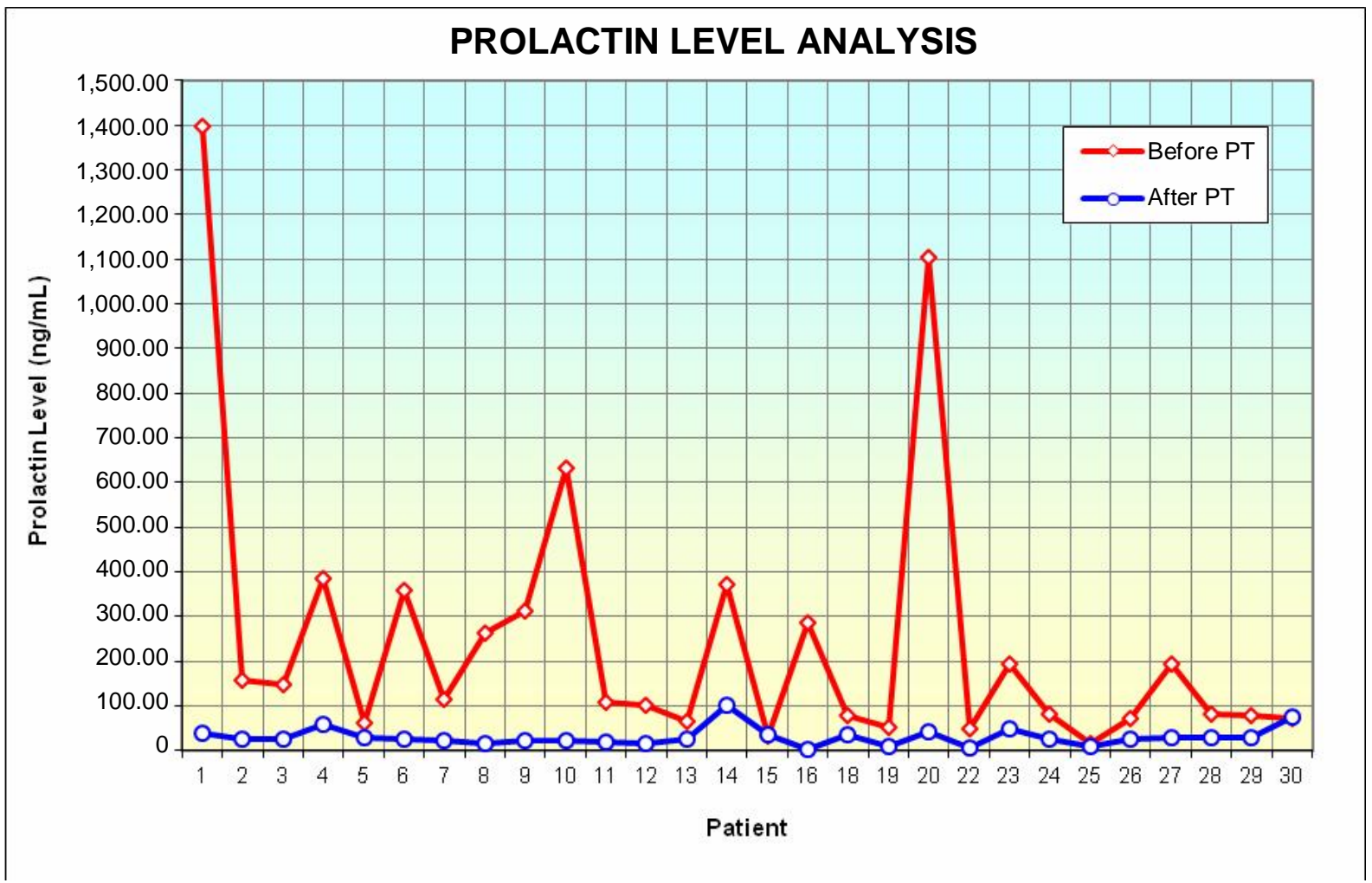

Figure 1: Prolactin levels determined prior and post PT across the entire study sample.

Referent intervals: female population, $2-30 \mathrm{ng} / \mathrm{mL}$; male population, $2-20 \mathrm{ng} / \mathrm{mL}$.

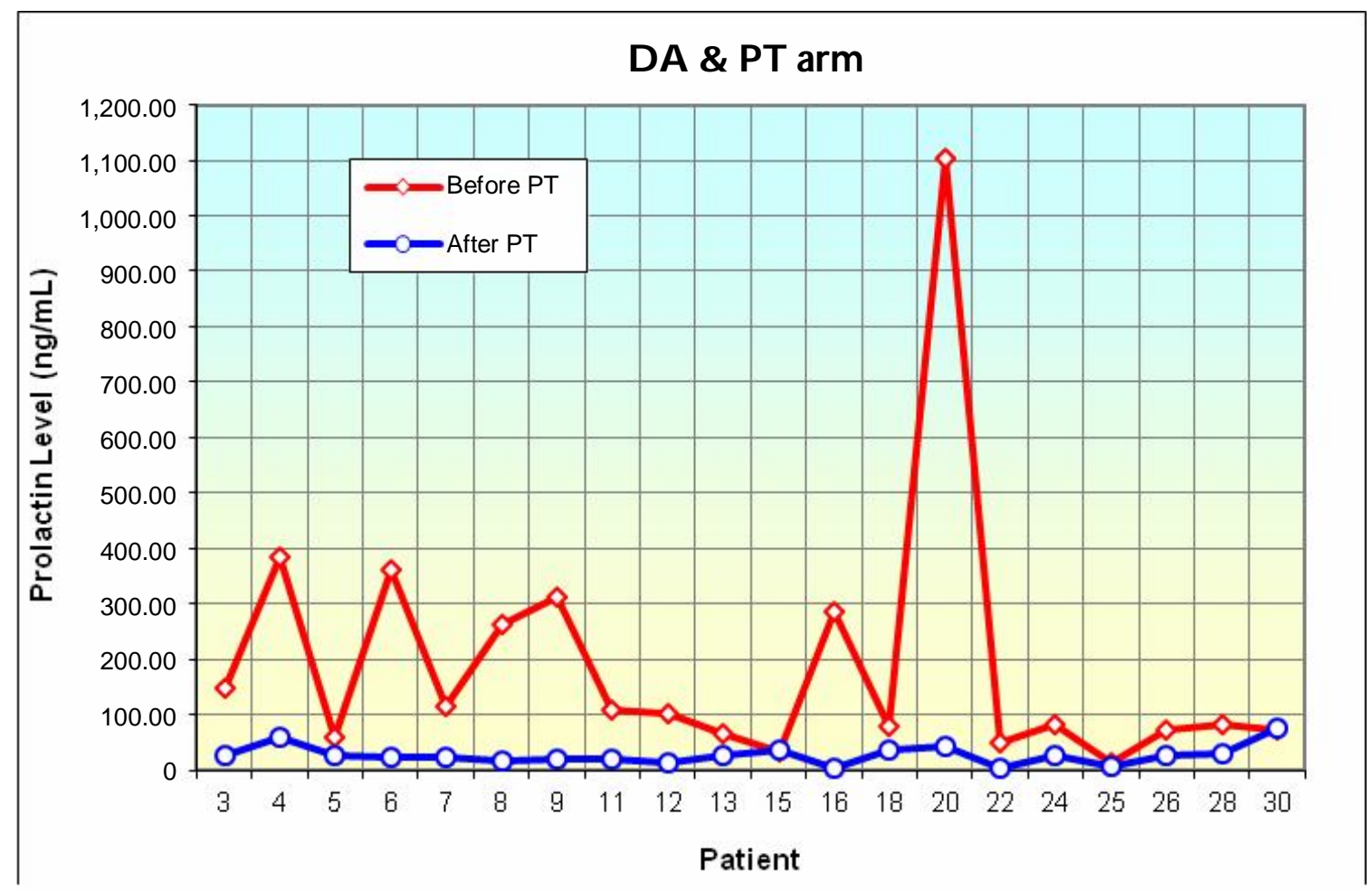

Figure 2: Prolactin levels witnessed in the DA\&PT arm prior to and following PT

Referent intervals: female population, $2-30 \mathrm{ng} / \mathrm{mL}$; male population, $2-20 \mathrm{ng} / \mathrm{mL}$.

The assumed PT efficiency was corroborated by testing the hypothesis on PRL means prior to and following PT, done at the significance levels of $5 \%$ and $1 \%$. The above statement applies also for $0.1 \%$-significance level, as corroborated by an adequate hypothesis testing. For visual display of the results obtained with both lines of treatment (DA and PT), please refer to Figure 2.

Group 2: PRL changes witnessed in the PT arm 
Out of the 30-patient pool, 8 were unable to tolerate side-effects arising on the grounds of DA treatment, so that after a short trial period they ceased taking the prescribed medication and resorted to PT only. Prior to the PT launch, some of the patients in this arm had the highest PRLs of them all, so that the decrease in PRLs seen following phytotherapy was far more striking than in the remaining study patients. PRL decrease witnessed following PT only (illustrated by Figure 3) proves, without a shred of doubt, the efficiency of herbal remedies in diminishing macroprolactinoma secretory potential. In 7 of these patients, PRL fell beyond $50 \mathrm{ng} / \mathrm{mL}$, while in one female patient (designated as patient No 14), who chose to undergo PT until the pre-scheduled surgery, a 4 month-lasting PT resulted in PRL drop from 373 to $102 \mathrm{ng} / \mathrm{mL}$.

In this arm as well, descriptive statistics pointed towards significant differences in pre- and post-PT PRL means, viewed as the primary outcome measure. Prior to the PT commencement, the PRL mean of $384.3 \mathrm{ng} / \mathrm{mL}$ was registered, while following PT completion the value in reference equalled to $38.0 \mathrm{ng} / \mathrm{mL}$. The difference in pre- and post-PT PRL means equals to $346.3 \mathrm{ng} / \mathrm{mL}$, disclosing a $90.1 \%$-PRL decrease.

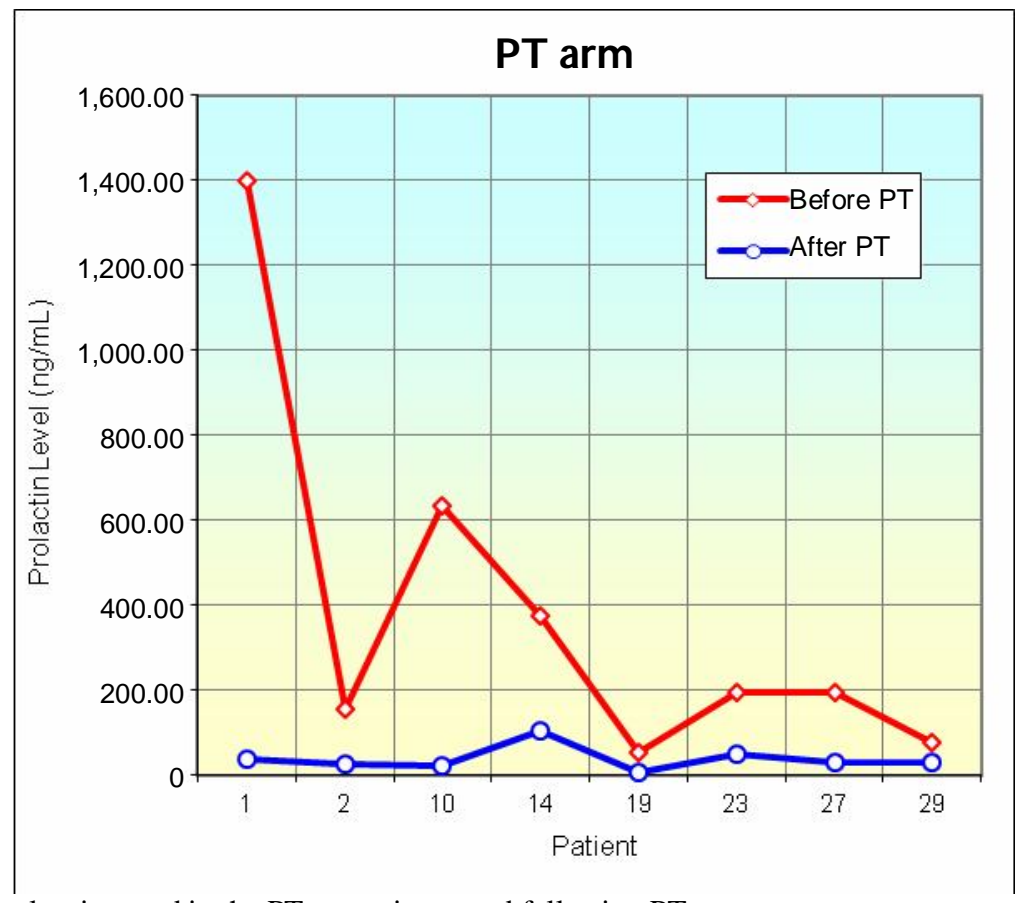

Figure 3: Prolactin levels witnessed in the PT arm prior to and following PT

Referent intervals: female population, $2-30 \mathrm{ng} / \mathrm{mL}$; male population, $2-20 \mathrm{ng} / \mathrm{mL}$.

The assumed PT efficiency was corroborated by testing the hypothesis on PRL means prior to and following PT, done at the significance level of $5 \%$. The above statement applies also for $1 \%$-significance level, as corroborated by an adequate hypothesis testing. For visual display of the results discussed above, please refer to Figure 3.

\section{Comparison of PRLs registered in DA \& PT vs PT arm}

Comparative analysis of PRLs registered in the patients constituting the DA \& PT versus those registered in the patients constituting the PT arm, aims at comparing the outcomes of these two distinctive lines of treatment. Testing of hypotheses on differences in PRL means completed at 5\%-significance level, failed to reveal any statistically significant changes in pre-treatment PRL means registered in these two arms. In other words, it can be stated with a 95\%-certainty (and a risk of error of 5\%) that PRL means seen across these two patient arms prior to treatment did not significantly differ from each other. From the statistical standpoint, this means that both patient arms had even "starting positions" allowing for a substantiated evaluation of the efficiency of different therapeutic modalities.

However, comparative analysis of mean PRLs seen in PT vs DA \& PT arm corroborate the hypothesised efficiency of both treatment modalities. By virtue of testing the hypothesis on differences in PRL means, done at 5\%-significance level, it was proven that a statistically significant difference in PRLs obtained across the PT arm and those obtained across the AD $\&$ PT arm is virtually non-existent. To rephrase, one can be 95\% certain (risk of error $=5 \%$ ) that mean PRLs obtained across these two distinctively treated arms following treatment completion do not differ to a statistically significant extent. Given the small number of PT arm members ( $n=8$ only), one should not jump into conclusions on the sole virtue of this line of treatment, but rather strive to repeat the analysis once a more substantial, i.e. a statistically more representative number of patients/data is at hand.

\section{Discussion}

The influence of PT on prolactin level (PRL)

Roughly $70 \%$ of raw materials utilised by pharmaceutical industry are of biological, i.e. natural origin, the vast 
majority of them thereby being isolated from plants (Kovačević, 2000; Pekić, 1983). Contemporary medicine stemmed from the traditional, folk medicine, but its huge development witnessed throughout the $20^{\text {th }}$ century had eventually led to a marked loss of interest in herbal preparations, so that their production had been pursued only within narrow circles of laymen heavily dependent on tradition, old ways and recipes passed across generations. Lack of medical knowledge among herbal remedy manufacturers and disregard shown by medical profession are the reasons why, until recently, the studies of the therapeutic efficiency of herbal remedies had been so scarce and rare. In the last twenty years, major changes in these views and attitudes have been witnessed, resulting in a re-evoked interest in plant-based preparations. Within this context, not only laymen, but medical profession as well, have decided to review their standpoints and attitudes towards this line of treatment. As a result, a more thorough and more in-depth research of therapeutic potentials of certain remedial plants and plant mixtures has been performed. Especially in the last few years, an ever-growing number of studies has proven that remedial plants deserve to be considered as a valid therapeutic option in a number of conditions (Block and Mead, 2003; Kanowski and Hoerr, 2003; Yale and Liu, 2004). The fact that our study patients had previously been treated in-ward specialised healthcare facilities, gives an additional rise to the significance of our results.

After being diagnosed with macroprolactinoma, the entire group of 30 patients enrolled into this study started their treatment with dopamine agonists (DAs) as the first-choice drugs when it comes to macroprolactinoma pharmacotherapy. In case of our patients, the therapy was unanimously started with the most commonly used dopamine agonist, bromocriptine. The results of DA treatment had been poor and, except for one patient, boiled down to a modest PRL decrease. As a rule, in case of resistance to therapy or in case of a poor response, treating physicians decide to switch their patients to cabergoline. However, it should be noted that our study sample consisted of patients coming from the former Yugoslavia (most of them from B \&H and Croatia, two from Serbia and one from Macedonia). Throughout the study period (2005-late 2009), the only DA authorised for market release in these countries was bromocriptine, while other DAs could be obtained exclusively from abroad and had to be paid out of the patients' pockets. Therefore, only 3 of our patients got to be treated with cabergoline, which might explain the poor results of pharmacotherapy administered to our study group prior to, or concurrently with, phytotherapy. In addition to pharmacotherapy, 13 patients included into this study underwent surgery, 4 of them even twice. However, due to various reasons, not a single patient had his/her tumour fully removed, so that, over time, tumour residua had re-grown into a macroprolactinoma. Facing such unsatisfactory results, the patients in reference decided to make PT arrangements.

The results achieved in the DA \& PT arm as well as those obtained in the PT arm, demonstrate, beyond doubt, a favourable effect of herbal remedies on macroprolactinoma secretory potential in terms of its putdown. A special attention should be paid, and a special credit given to, the results achieved in 8 patients undergoing PT only, since they pose as a role model for the efficiency analysis of PT chosen to be not merely an adjuvant, but the "number one" macroprolactinoma therapy. The results obtained within this frame suggest that in some macroprolactinoma patients satisfactory PRL regulation can be achieved by virtue of herbal remedies as the first choice treatment. Following the PT completion, a similar percentage of PRL decrease was seen across the DA \& PT arm as well.

Comparison of PRL means registered prior to and following PT failed to reveal any statistically significant differences in the aforementioned outcome measure between the PT and the DA \& PT arm. It should be noted, however, that the decrease in PRLs that DAs should be given credit for, seen across the AD \& PT arm, was only modest, the prescribed DA dose thereby being more in function of maintenance of the achieved PRL levels then in function of their further diminishment. Truth is that DAs had succeeded in their mission and had prevented PRLs from further rise, but had never managed to reduce them beyond the values displayed on Figure 2, so that further decrease in PRLs, seen across this arm post treatment, should apparently be attributed to the PT introduced later on.

Concurrent PT and DA administration failed to reveal any synergy between the two. The action mechanism of pharmaco-active plant ingredients remains unclear; however, possible mediation of this suppression of PR secretion by D2 receptors can be definitely ruled out.

One of the research tracks to be pursued in further course is the quest for plausible explanation of PT impact on macroprolactinoma secretory potential. By all means, the explanation of this mechanism shall uncover yet unknown paths of PR synthesis and release.

Noteworthy, data on PT impact on macroprolactinoma secretory potential presented herein, are genuine and the very first in these parts and, to the best of our knowledge, in the world as well. The available literature offers no similar studies, so that the comparison to the experience of other authors is rendered virtually impossible.

\section{Contraindications for and the length of PT}

DA-treated patients are forced to a multiyear therapy, but the use of DA drugs has its limitations. Subsequent to a multiyear follow-up of patients taking DAs for a prolonged period of time, on June $26^{\text {th }} 2008$ the European Medicines Agency (EMEA) released the announcement that communicates new warnings and contraindications for DA use, in specific an increased risk of fibrosis, especially heart fibrosis, attributed to a chronic ergot alkaloid (a DA derivative) use (Pehaček and Sajdl, 1990). The revision in reference addressed two most commonly used DAs, i.e. bromocriptine and cabergoline, as well. In line with the EMEA recommendations, the maximal daily cabergoline dose is restricted to $3 \mathrm{mg}$, while that of bromocriptine should not surpass $30 \mathrm{mg}$ (EMEA; Europe, 2011). The highest daily bromocriptine dose used by one of our patients equalled to $40 \mathrm{mg}$.

The length of PT pursued by our patients spanned from 3 to 41 months, while 9 of them had been treated for 10 months or longer. Regardless of treatment duration, the patients had no contraindications for PT at all, nor did they experience any symptoms potentially attributable to phytotherapy. The earliest benefits of PT-induced PRL putdown were witnessed by female patients of childbearing age, in whom ovulation and normal menstrual cycle had been restored. What the authors find most encouraging and intriguing about their results is the fact that two female patients got pregnant while on PT and eventually gave birth to healthy children. Though counted into the study sample, data on these two patients were not 
included into the statistical analysis since PRL data were lacking due to pregnancy that occurred during the course of PT. Nevertheless, PR levels in these two patients must have been somewhere within the referent boundaries or just slightly above the upper normal; otherwise, getting pregnant would be virtually impossible. Prior to the PT commencement, both patients were treated with DAs. Once they got pregnant, the patients ceased to take DAs but continued PT throughout pregnancy. PT continuation throughout pregnancy was recommended for preventative reasons, since it is well known that estrogens, whose levels rise during pregnancy, play an important role in tumour development and growth by means of stimulating lactotrophic cell hyperplasia. This goes especially for large, rapidly-growing tumours, known to be more prone to estrogenic stimulation (Mah and Webster, 2001). Nevertheless, having regard to the fact that our experience in this matter currently boils down to two female patients only, additional data on the impact of herbal remedies during pregnancy should be gathered on a fairly large, more representative sample, so as to be able to obtain more reliable and substantiated results on the effectiveness and safety of phytotherapy during pregnancy. The ultimate PT length is not precisely defined; the same applies also for the optimal length of PT administration. To our knowledge, PT duration needed for its benefits to become apparent, is dependent on pre-PT prolactin levels, but the first therapeutic results should be expected 3-5 months following the PT commencement.

The significance of this study lies within the fact that it clearly demonstrates the possible use of PT in macroprolactinoma patients to the effect of PRL regulation. Due to such therapy, the following benefits can be attained:

- $\quad$ The possibility of combined PT + DA treatment;

- $\quad$ The possibility of preventative PT treatment of pregnant women suffering from macroprolactinoma; otherwise, a tumour growth during pregnancy, facilitated by hyperestrogenism, might be experienced; and, bottom line,

- In carefully selected patients, PT might just as well prove itself as the first choice therapy of macroprolactinoma.

\section{Conclusion}

The results of this five-year study suggest the possibility of phytotherapeutic treatment of macroprolactinoma patients to the effect of hyperprolactinaemia regulation. This line of treatment had proven itself worthy of being not merely an adjuvant, but, in some patients, the first choice macroprolactinoma therapy. This claim is particularly based on the encouraging results obtained across our PT arm. Future research should attempt to demonstrate the efficiency of PT as the first choice therapy capable of downsizing macroprolactinoma-induced prolactin hyper-secretion, while the results obtained with this line of research should be compared against the current notions on DA efficiency.

\section{Acknowledgement}

We are most indebted to all of our patients for putting their trust in us and entrusting us with their health. Without them and their faith in phytotherapeutic benefits and potentials, this study wouldn't be possible.

\section{References}

1. Asa, S.L. (1988). Tumours of the Pituitary Gland. In: Rosai, J., Sobin, H. Armed Forces Institute of Pathology, Washington D.C., third series, Fasc $22: 1-120$.

2. Braucks, G.R., Naliato, E.C., Tabet, A.L., Gadelha, M.R. and Violante, A.H. (2003). Clinical and therapeutic aspects of prolactinoma in men. Arq. Neuropsiquiatr., 61: 1004-1110.

3. Guyton, A.C. and Hall, J. E. (2003). Textbook of Medical Physiology, Philadelphia, Pennsylvania : W.B. Saunders Company : 846 - 857

4. Horvath, E. and Kovacs, K. (1992). Ultrastructural diagnosis of human pituitary adenomas. Microsc. Rec. Tech., 20: $107-135$.

5. $\quad$ http://www.emea.europa.eu/pdfs/human/press/pr/32239508en.pdf

6. Block, K.I. and Mead, M.N. Immune System Effects of Echinacea, Ginseng, and Astragalus: A Review. (2003). Integr. Cancer Ther., 2: 247-267.

7. Kanowski, S. and Hoerr, R. (2003).Ginkgo biloba extract EGb 761 in dementia: intent-to-treat analyses of a 24week, multi-centre, double-blind, placebo-controlled, randomized trial. Pharmacopsychiatry, 36: 297-303.

8. Kovačević, N. (2000). Pharmacognostic basics. Faculty of Pharmacy University of Belgrade: 35-212.

9. Levy, A and Lightman, S.L. (1993). The pathogenesis of pituitary adenomas. Clinical Endocrinology; 38: 559-7.

10. Lukić, P.B. (1993). Pharmacognostics. Faculty of Pharmacy University of Belgrade: 18 - 24.

11. Mah, P.M. and Webster, J. (2001). Hyperprolactinaemia: aetiology, diagnosis and management. Semin. Reprod. Med., 20: 365-374.

12. Pehaček, Z. and Sajdl, P. (1990). Ergot alkaloids: Chemistry, biological effects, biotechnology. Prague Academy:

$1-42$.

13. Pekić, B. (1983). Chemistry and technology of pharmaceutical products - alkaloids and ether oils. Faculty of Technology University of Novi Sad: 1-68.

14. Yale, S.H. and Liu, K. (2004) Echinacea purpurea Therapy for the Treatment of the Common Cold: A Randomized, Double-blind, Placebo-Controlled Clinical Trial.

Arch. Intern. Med., 164: 1237-1241. 